\title{
Enhancing apparel data based on fashion theory for developing a novel apparel style recommendation system
}

\begin{abstract}
Smart apparel recommendation system is a kind of machine learning system applied to clothes online shopping. The performance quality of the system is greatly dependent on apparel data quality as well as the system learning ability. In order to develop a better system, this paper proposes (1) to enhance apparel data quality based on fashion communication theories and (2) to use deep learning methods for machine learning. The novelty of the proposed methodology embodies two aspects, introducing a rich apparel knowledgebased data and new training models with deep learning driven approaches. The acquisition of new apparel data is supported by apparel visual communication and sign theories, as well as skilled experts with practical experiences on clothes and dressing. In machine learning, a two-step training model is proposed. The first step is to predict apparel ATTRIBUTEs from the raw image data through a multi-task Convolution Neural Network model. The second step is to learn apparel MEANINGs from predicted attributes through two separate classifiers, SVM and newly proposed LKF classifier. The testing results show that the prediction rate of eleven predefined MEANING classes can reach the range from $80.1 \%$ to $93.5 \%$ through LKF so that the proposed apparel data and training methods are effective. The two-step apparel learning model is applicable for advanced Menswear search and style recommendation systems.
\end{abstract}

Keywords: Apparel recommendation, Body, Style, Visual communication system, Apparel Data, Deep learning.

\section{$1 \quad$ Introduction}

In order to improve online shopping experiences and users' satisfactions, apparel recommendation systems that combine online apparel data with intelligent computing technologies have attracted research attentions. Traditionally, obtaining customers information and their purchasing history data is a prerequisite for developing recommendation systems. It suffers from the cold start due to the lack of historical data for new customers. Furthermore, recommending clothes that are similar to the previous liked ones may not work for the fashion products since people tend to seek style changes or even style revamp. With the growing Artificial Intelligence technologies, recent studies have started to develop more professional apparel recommendation systems. However, the domain knowledge of clothes and dressing is used fragmentally in various systems, such as dressing for bodies in Vuruskan et al. (2015)'s recommendation system, dressing for occasions in Liu et al. (2012) 's recommendation system and mix-and-match styling recommendation system developed by Vaccaro et al. (2016). 
This study aims to integrate the profound knowledge and theories of clothes, fashion and dressing into the development of high quality apparel datasets and intelligent learning models to support apparel-oriented smart recommendation systems. We introduce a new apparel data coding system and two datasets: ATTRIBUTE and MEANING, based on clothes communication and semiotic theories. The ATTRIBUTE data captures the visual elements of design such as lines, shapes, colours, patterns, materials, mix and matches etc. and the MEANING data captures the connotation meanings carried by visual clothes and outfits. We also develop a deep learning model that predicts apparel MEANINGs through the apparel images and ATTRIBUTEs. The proposed methodology above can enhance the quality of an apparel recommendation system.

The remaining of this paper is organized as follows: section 2 reviews existing apparel data and recommendation systems. Section 3 investigates the theory of clothes, menswear and fashion. Section 4 introduces the definition, construction and collection of newly developed apparel datasets based on visual communication theories. The following section details the methods of training apparel datasets and testing results. Section 6 draws conclusions and future works.

\section{Related Work}

Current studies have been focusing on the apparel data revolution in recommendation system development. The earlier systems excavated user's profiles and bought/liked history data and recommend new products based on images similarity. A raising research concern was that apparel recommendations should consider both clothing and dressing issues. Occasion-oriented recommendations extracted clothes image features and labelled it through crowdsourcing method (Liu et al. 2012). Customer profile and behavioural data were replaced by occasion data offered by online crowdsourcers. Moreover, some researchers focused on outfit images designed by domain experts for developing mix-and-matches recommendation systems (Jagadeesh et al. 2014; Kalantidis et al. 2013). The newly developed intelligent systems started to integrate with clothing and fashion knowledge such as providing recommendations based on fashion DNA, styling, body dressing (Bracher et al. 2016; de Barros Costa et al. 2017; Landia 2017; Vaccaro et al. 2016; Vuruskan et al. 2015).

Applied methods for developing intelligent recommendation systems can be divided into two parts, feature extractions and classifications. Current research has showed the abilities of using computer to detect texture (Kim et al. 2013), outline shape (Cheng and Liu 2008) and some local features (Iwata et al. 2011) such as the Scale Invariant Feature Transform (SIFT). Automatic extractions could generate a large volume of image-based feature data, but it may be less accurate to represent essential apparel characteristics. It is necessary to bridge the connections between image features and apparel design elements. Regarding to classifications, the conventional machine learning algorithms are the dominant methods such as Neural Networks and Support Vector Machine. This study is going to develop a deep learning 
model to extract design attributes from raw images and make classifications using separate classifiers.

\section{The Theories of Clothes, Menswear and Fashion}

The understanding of clothes particular on Menswear fashion involves multiple disciplines such as design, sociology, semiology, economics and cultural studies. According to the fashion theory by Barnard (2007) and Men's fashion theories by McNeil and Karaminas (2009), we found important theories and knowledge to support apparel data and recommendation system development. The new viewpoint is that clothing is a kind of language for people to communicate meanings. For example, Barnard elaborated the relationship of fashion, clothing, communication and meaning in his book named Fashion as Communication (Barnard 2002). This theory explained what the apparel might be for the wearer, why people wear clothes and how people use the meanings of clothes for effective communication.

\subsection{An overview of Fashion theory and Men's fashion theory}

The context of wearing clothes was discussed in some fashion theories such as Clothes and the Body, Style and Identity, and Clothes and Communication. Firstly, the body could bring meanings to dress through adornment. In dressing male bodies, Odile Blanc stated that dress amplified the body in particular ways and was part of male power strategies (Blanc 2002). The transposition of the clothes' attributes and the feelings that it invokes is not merely the effect of the overall apparel as a casing, but of the body itself (Karaminas 2009). Dress serves as a visual metaphor for identity.

The message of clothes implies the identity regarding sex and gender, social class, ethnicity and race, as well as culture and subculture (Barnard 2007). The unique identity in men's style is the Masculinity. It is constructed with certain social background and changing with time, as Peter McNeil indicated that the meanings of men's dress are transformed in different cultural and historical contexts (McNeil and Karaminas 2009). Tim Edwards addressed men's fashion and masculinity through consumer society (Edwards 2016). He emphasized the significance of sign value which is the meaning attached to commodities.

Rouse in Understanding Fashion indicated that the important function of clothes is the communication (Rouse 1989). Barnard in Fashion as Communication stated that clothing is a non-verbal communication medium where one person as the wearer intends to send messages and another person as the spectator/viewer receives the messages (Barnard 2002). The clothes language and communication theory could fit the men's fashion. These arguments specifically explained the message of men's style and its communication value. 


\subsection{Embedding the apparel communication theory into knowledge-based recommendation system development}

Apparel communication is a complex system which occurs at two levels: clothes-body communication and wearers-viewers communication (Fig.1). In level one, wearers themselves express meanings through the visual perception of clothes on their body, with visual and touch feelings. In level two, viewers perceive meanings through the visual perception of clothes on wearer's body without touch feelings. The overlap area of meanings in both levels evaluates the quality of apparel communication. Hence, supporting meanings to be communicated correctly is the target that apparel recommendation system is trying to achieve.

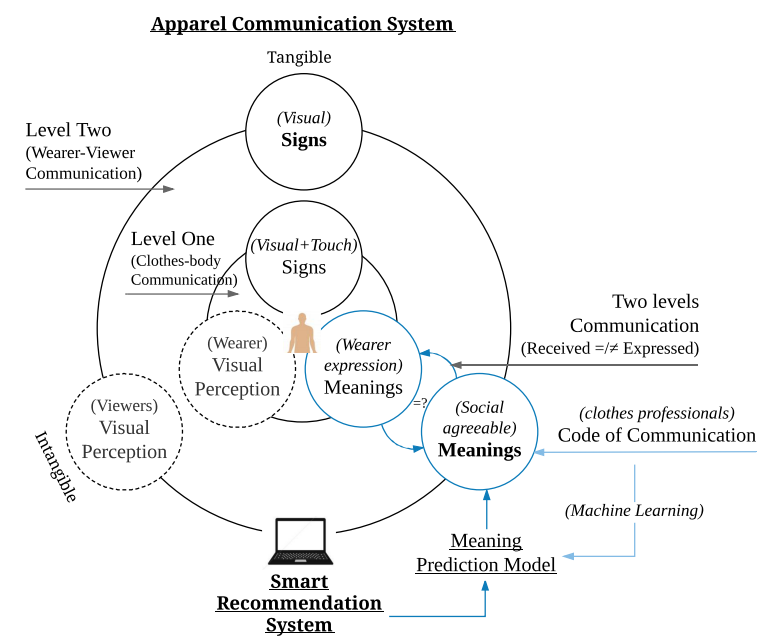

Fig. 1. The relationship of apparel communication system and smart recommendation system

The development of an apparel recommendation system needs to focus on apparel meaning prediction model, aiming to effectively estimate meanings that could match up viewers' perceived with wearer's intended to express. In fact, the code to support apparel communication is better known by clothes professionals since these people have particular knowledge, skill and experiences of clothes and dressing. Thus the method of developing meaning prediction model is using machine learning and deep learning algorithms to train a learning model based on apparel communication theory and practical expert knowledge.

\section{$4 \quad$ Knowledge-encoded apparel data}

\subsection{Data definition, construction and collection}

Figure 2 illustrates an apparel communication network composed of three major components, including apparel signs, denotation meanings and connotation meanings. Signs of apparel refer to visual elements of line, shape, volume, drape and other ele- 
ments in the listed. The first order of communication is between visual signs and denotation meanings. It commonly indicates obvious or literal meanings of apparel, but this study introduces a second order that indicates the semantics of design. The second order of communication is between denotation and connotation meanings. It generates meanings of mental (or perceptual) feelings that may associate with body, occasions, design genres or personal preferences. The second order of meanings is finally applied for communication.

The structure of apparel datasets is connected with the clothes communication networks. Apparel signs are composed by original apparel image data. Denotation meanings indicating apparel itself are belonged to the ATTRIBUTE dataset, and connotation meanings given by people outside of apparel are filled to the MEANING labelling dataset.

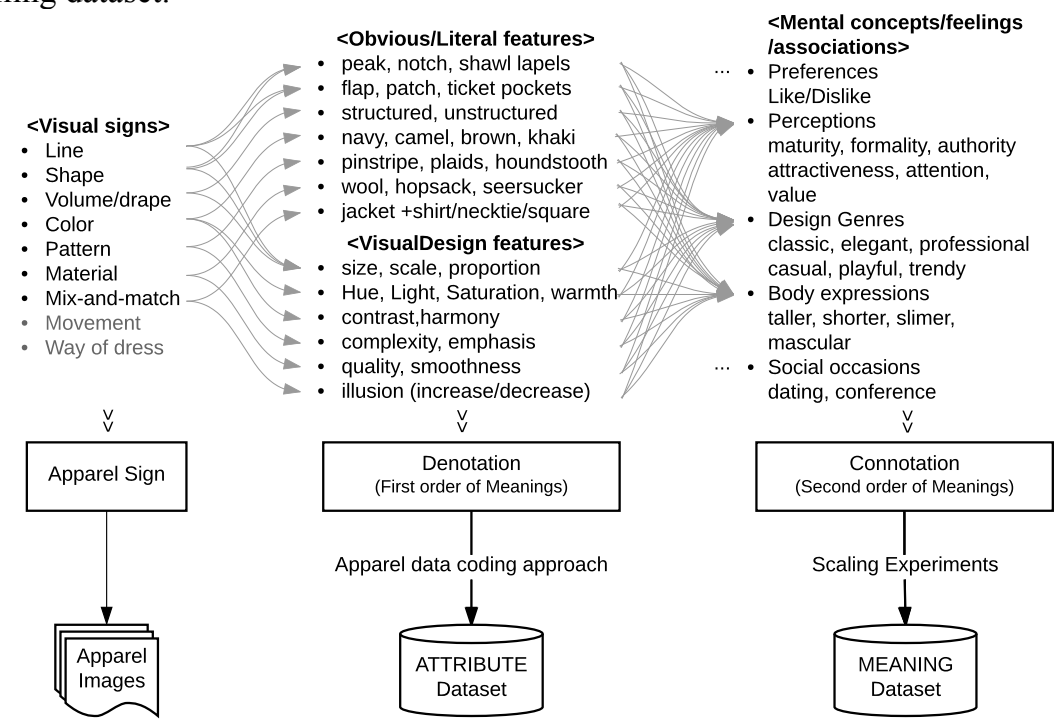

Fig. 2. Apparel communication networks and data definitions

ATTRIBUTE dataset. The ATTRIBTUE data captures the first order of meanings, which is the denotation meanings generated from apparel itself, such as lines, shapes or colours. It nominally denotes the obvious and literal meanings of clothes, such as lapel shape is denoted as peak, notch or shawl. The deeper denotative meaning involves the design of lines, shapes and colours, such as the proportion of lapel shapes. More design features, such as the contrast, harmony, illusion, emphasis and complexity (Fiore 2010) could be adopted to denote deep attributes. An extended semantic attribute data has been defined which denotes both natural and design meaning derived from the internal of apparel.

All of the identified visual features of apparel signs were classified into 25 categorical variables (e.g. proportion) and 20 ordinal variables (e.g. buttons number) based on their properties. For coding, for instance, the ration of a Jacket width/length as one of 45-dimensional features can be encoded as $1=$ 'wide/short', $2=$ "narrow/short', 
$3=$ 'narrow/long', and 4='wide/long' from the subcategories. All samples (220s) were encoded into SPSS 24.0 system manually according to each sample's product descriptions on its purchasing website. After eliminating samples with missing features, a number of 200 samples went into the final dataset.

MEANING dataset. The MEANING data is identified as the second order of meanings, connotation meanings that are generated outside of apparel by people, such as wearers, viewers or designers. It denotes the feelings, thoughts, beliefs and desires of clothes based on mental concepts. General speaking, peak lapel feels formal. Peak lapel with a wider shape and higher position of gorge could formulate a muscular chest look. This study presents connotation meanings by perceptual feelings (style genres, e.g. elegant) and body meanings (to indicate body shapes). These identified meanings will be used to label apparel samples. As mentioned earlier, the second order of meaning is given by people. Therefore, it is necessary to decide which people could contribute to those meanings. The clothes professionals are qualified as skilled people. This group will contribute to data collection in labelling experiments.

The MEANING data was collected through labelling tasks by clothes experts. A 11-class set of style MEANING adjectives was employed as predefined labels including style genres of Casual, Trendy, Professional, Elegant, Classic and Playful, as well as body corrections for Square (broad all over), Circle (chubby all over), Triangle (soft round middle), Invert Triangle (broad shoulders slim waist) and Slim (straight up and down). The annotators were five commercial stylists with at least two-year working experience in a large retail sector. The labelling adjectives of apparel samples were recorded into SPSS 24.0 in accordance with ATTRIBUTE data.

\subsection{Data evaluations}

The quality of the proposed two apparel datasets has been evaluated by several statistics and content-based assessments. We employed Internal Consistency and TestRetest assessments to evaluate the reliability. Exploratory and confirmatory factor analyses measured the validity through statistical correlations of data. A panel of experts inspected the validity through the content structure. Statistics-based assessments confirmed that the element correlations in statistics are consistent with real connections. The feedback of content-based reviews by domain experts supported current structure and provided additional adjustments for further developments.

\section{Development of apparel learning models for recommendation system}

\subsection{Apparel learning model design}

In an apparel communication system, meanings are transmitted through three layers including the apparel sign, denotation meanings and connotation meanings. The last layer, connotation meanings are the final output of communications. Logically, the 
learning model of connotation meanings could base on the features extracted from raw apparel Images or features represented by ATTRIBUTEs. Therefore, we essentially develop a deep learning model to extract image-based features that are discriminative to ATTRIBUTEs and MEANINGs and make classifications through a multitask Convolutional Neural Network (CNN). The further training model is using predicted ATTRIBUTEs from the output of CNN to learning the MEANINGs, with two separate classifiers SVM and a newly proposed LKF.

A deep CNN model. We proposed a deep CNN structure which serves as a multi-task model considering all of the three levels of predictions, including style genres, denotation attributes, and body shapes. The key configurations of the proposed deep learning model are summarized in Figure 3. The proposed CNN model is composed of five convolutional layers, five pooling layers and two fully connected layers. The design from the first convolutional layer to the last pooling layer serves for the feature learning. Each convolution level has two layers in the same size, e.g. Conv1 has Conv1_1 and Conv1_2 in the size of $160 \times 128$. The max-pooling layers between two levels make the size shrink to half of the original size. After Conv5, all of the patches are concatenated and mapped to two layers of fully connection, Fc6 and Fc7. The resultant feature space is in $X$ (apparel) $=\left[\mathrm{x}_{\mathrm{n}}\right] \in \mathbb{R}^{N \times 4096}$, where $1, \ldots, \mathrm{n}, \ldots N$ indexes the training samples. The design from fully connected layers to the final outputs serves for classifications. There are two types of objective functions, Cross Entropy for $Y$ (style genre) and Y(body shape), and Norm-2 distance for Y(attribute).

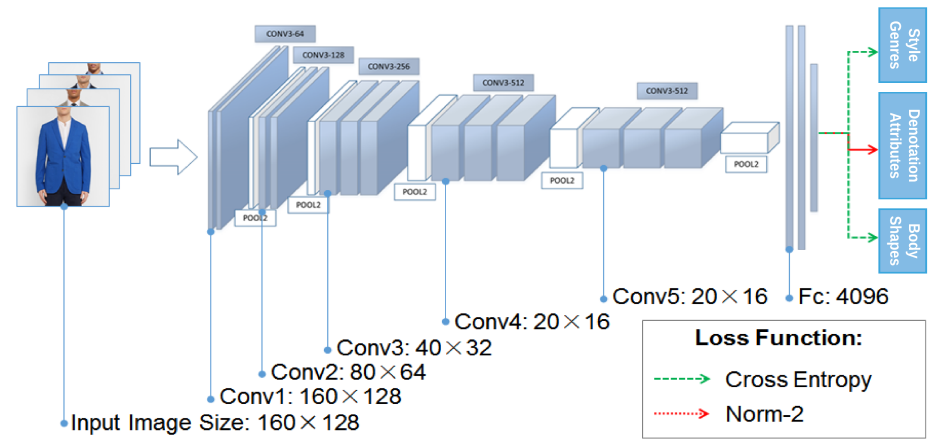

Fig. 3. Key configurations of the proposed deep learning model, Conv: convolution layers; Fc: fully connection layers.

MEANING prediction model. Secondly, we train apparel MEANING prediction model using predicted apparel ATTRIBUTEs from the outputs of CNN model. Since $\mathrm{CNN}$ feature is obtained by learning the multi-task loss regarding apparel attributes, style genres and body shapes, the predicted attributes may have been adjusted for the loss of style genres and body shapes prediction. Therefore, using the predicted attributes from the output of CNN model could further benefit the style genres and body shapes predictions. 
Since the deep learning model is based on a multi-task loss, empirically, the performance of each individual task may have been sacrificed for the sake of balanced overall loss. Therefore, the separate SVM classifiers are introduced for predictions with extracted visual features from CNN model. However, the proposed SVM classifier may encounter the degradation due to the small training size. Because our evaluation is based on a highly random cross-validation scheme, the maximum number of training images is 180 , and the samples of each label can be very unbalanced. The weak classes with few samples may be completely sacrificed for the denser ones. In order to mitigate the unbalanced prior, we propose a distribution based classification algorithm that is named as Later Kernel Fusion (LKF). Instead of learning the class boundaries, LKF focuses on maximizing the likelihood function between the test samples.

\section{$5.2 \quad$ Results}

Above proposed apparel learning approaches have been implemented through Python scripts. We feed the Image, ATTRIBUTE and MEANING three parts of data as the inputs and outputs to train models. The model testing results are demonstrated in table 1. The average prediction rate of 11 predefined classes is $74.9 \%$ in the deep CNN model. To combine with the conventional SVM classifier is less effective. In comparison, the average predictability with the newly proposed LKF classifier is $9.4 \%$ higher than the first introduced CNN model. The MEANING prediction model further improved the performances of eleven classes by introducing the predicted ATTRIBUTEs in CNN model as feature data. The most significant increases are in the class of 'Circle' from the CNN model (76.4) to the CNN model with LKF (84.4) and finally to the MEANING prediction model with LKF (90.4).

Table 1. Model training and testing results

\begin{tabular}{|c|c|c|c|c|c|c|c|c|c|c|c|c|}
\hline & \multicolumn{11}{|c|}{ Predictability of Classes (style genres \&body shapes) (\%) } & \multirow[b]{2}{*}{ Mean } \\
\hline & Casual & Elegant & Playful & $\begin{array}{l}\text { Profes } \\
\text { fes- } \\
\text { sional } \\
\end{array}$ & Trendy & Classic & Circle & $\begin{array}{l}\text { Invert } \\
\text { triangle }\end{array}$ & Triangl & Square & Slim & \\
\hline CNN Model & 72.8 & 74.5 & 69.4 & 75.8 & 80.5 & 72.2 & 76.4 & 71.8 & 67.7 & 79.6 & 82.8 & 74.9 \\
\hline CNN Model (with SVM) & 72.9 & 72.6 & 70.1 & 76.2 & 77.5 & 70.7 & 75.8 & 69.5 & 64.4 & 79.7 & 79.2 & 73.5 \\
\hline CNN Model (with LKF) & 82.4 & 83.8 & 77.9 & 84.6 & 91.2 & 85.7 & 84.4 & 82.5 & 79.6 & 86.4 & 89.2 & 84.3 \\
\hline MEANING Prediction Model (with SVM) & 79.6 & 85.1 & 76.9 & 84.3 & 91.8 & 86.9 & 88.4 & 84.5 & 83.8 & 91.4 & 93.2 & 86.0 \\
\hline MEANING Prediction Model (with LKF) & 84.5 & 85.4 & 80.1 & 88.5 & 92.4 & 87.1 & 90.4 & 86.3 & 87.8 & 92.6 & 93.5 & 88.1 \\
\hline
\end{tabular}

In feature extractions, apparel design attributes are recognisable from images through deep learning method, and the recognised apparel attributes are the most effective feature representations to adapt the tasks of apparel meaning classifications. In classifications, the proposed LKF algorithm is more effective than the baseline SVM in both CNN model and MEANING prediction model. Overall, the proposed MEANING prediction model with LKF classifier reached the best performance with an average prediction accuracy of $88.1 \%$. This is a highly effective and useful model for prototyping apparel recommendation systems. 


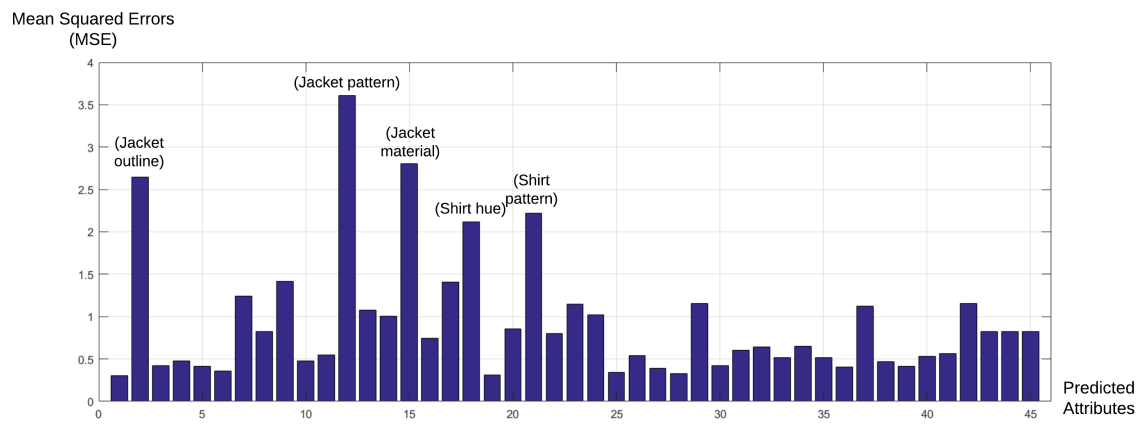

Fig. 4. Mean squared errors of predicted attributes

The prediction of apparel attributes is made by the regression output of the CNN model. Figure 4 illustrates the Mean Squared Errors (MSE) of overall predicted attributes, which estimate the average of the squares of the prediction errors. This is an important parameter to measure the performance of regression model. The value of MSE closer to zero means the higher predictability of apparel attributes. In figure 4 , the majority apparel attributes are predictable with small errors (MSE $<1.5)$. The predictions on five attributes are less accurate with the relatively higher MSE, including Jacket outline, jacket pattern, jacket material, shirt hue and shirt pattern. This is caused by the unbalanced distribution of samples in these high-dimensional attributes. Some features due to lack of observing samples are unlikely to be predicted.

\section{Conclusion}

Knowledge-based apparel recommendation systems could be achieved through the newly proposed apparel datasets and modelling methods. The ATTRIBUTE prediction model can be used for Menswear advanced search system that allows natural and design features to be filtered, and the MEANING prediction model can be used as a Menswear style recommendation engine that distinguishes online clothes by specific style genres or body shapes. These models are superior to other models in recognising in-depth clothes features and connotative meanings like the trained style professionals. Whereas, it currently only applicable to the Menswear category. In the future, the proposed methods could be examined with a larger size of sample data, a wider category of clothes, more predefined classes regarding apparel meanings, e.g. dandy, body colours, as well as occasional meanings.

\section{References}

Barnard, Malcolm (2002), Fashion as communication (Psychology Press).

--- (2007), Fashion theory: A reader (Routledge).

Blanc, Odile (2002), 'From Battlefield to Court: The Invention of Fashion in the Fourteenth Century', Encountering Medieval Textiles and Dress (Springer), 157-72. 
Bracher, Christian, Heinz, Sebastian, and Vollgraf, Roland (2016), 'Fashion DNA: Merging Content and Sales Data for Recommendation and Article Mapping', arXiv preprint arXiv:1609.02489.

Cheng, Ching-I and Liu, Damon Shing-Min (2008), 'An intelligent clothes search system based on fashion styles', Machine Learning and Cybernetics, 2008 International Conference on (3: IEEE), 1592-97.

de Barros Costa, Evandro, et al. (2017), 'Understanding and Personalising Clothing Recommendation for Women', in Álvaro Rocha, et al. (eds.), Recent Advances in Information Systems and Technologies: Volume 1 (Cham: Springer International Publishing), 841-50.

Edwards, Tim (2016), Men in the Mirror: Men's Fashion, Masculinity, and Consumer Society (Bloomsbury Publishing).

Fiore, Ann Marie (2010), Understanding Aesthetics for the Merchandising and Design Professional (New York: Fairchild Books).

Iwata, Tomoharu, Wanatabe, Shinji, and Sawada, Hiroshi (2011), 'Fashion coordinates recommender system using photographs from fashion magazines', IJCAI Proceedings-International Joint Conference on Artificial Intelligence (22: Citeseer), 2262.

Jagadeesh, Vignesh, et al. (2014), 'Large scale visual recommendations from street fashion images', Proceedings of the 20th ACM SIGKDD international conference on Knowledge discovery and data mining (ACM), 1925-34.

Kalantidis, Yannis, Kennedy, Lyndon, and Li, Li-Jia (2013), 'Getting the look: clothing recognition and segmentation for automatic product suggestions in everyday photos', Proceedings of the $3 \mathrm{rd}$ ACM conference on International conference on multimedia retrieval (ACM), 105-12.

Karaminas, Vicki (2009), 'Übermen: Masculinity, Costume, and Meaning in Comic Book Superheroes', in Peter McNeil and Vicki Karaminas (eds.), The Men's Fashion Reader (Berg Publishers).

Kim, Donghyun, Kwon, Young-Bin, and Park, Jaehwa (2013), 'A scoring model for clothes matching using color harmony and texture analysis', Graphics Recognition. New Trends and Challenges (Springer), 218-27.

Landia, Nick (2017), 'Building Recommender Systems for Fashion: Industry Talk Abstract', Proceedings of the Eleventh ACM Conference on Recommender Systems (ACM), 343-43.

Liu, Si, et al. (2012), 'Hi, magic closet, tell me what to wear!', Proceedings of the 20th ACM international conference on Multimedia (ACM), 619-28.

McNeil, Peter and Karaminas, Vicki (2009), The Men's Fashion Reader (Berg Publishers).

Rouse, E (1989), Understanding Fashion (Oxford: BSP Professional Books ).

Vaccaro, Kristen, et al. (2016), 'The Elements of Fashion Style', Proceedings of the 29th Annual Symposium on User Interface Software and Technology (ACM), 777-85.

Vuruskan, Arzu, et al. (2015), 'Intelligent fashion styling using genetic search and neural classification', International Journal of Clothing Science and Technology, 27 (2), 283-301. 